\title{
TRANSIENT WAVE ANALYSIS OF AN ANTIPLANE CRACK INTERACTION WITH HALF-PLANE BOUNDARY
}

\author{
CHIEN-CHING MA and YI-SHYONG ING \\ Department of Mechanical Engineering, National Taiwan University, Taipei, Taiwan 10617 . \\ Republic of China
}

\begin{abstract}
The transient problem of a traction free half-space containing a subsurface semi-infinite crack subjected to dynamic antiplane loading on the crack faces has been investigated to gain insight into the phenomenon of the interaction of stress waves with material defects. A specific loading condition, namely a pair of concentrated point loadings applied on the crack faces, is considered in detail. The transient solutions are determined by superposition of a fundamental solution in the Laplace transform domain. The fundamental solution to be used is the problem for applying exponentially distributed traction in the Laplace transform domain on the crack faces. The exact closed form transient solutions of dynamic stress intensity factors are obtained and are expressed in a very simple formulation in this study. These solutions are valid for an infinite length of time and have accounted for the contributions of an infinite number of waves. Numerical results of dynamic stress intensity factors are obtained which indicate that the major contributions are due to the first few waves. Copyright (C 1996 Elsevier Science Ltd
\end{abstract}

\section{INTRODUCTION}

The difficulty in determining the transient stress field in a cracked-elastic body subjected to dynamic loading is well known. A pre-existing crack inside the medium would disturb the propagation waves and make the theoretical analysis much more difficult than in an homogeneous medium. A considerable amount of research has been directed towards the solution of problems involving the interaction of stress waves with the crack and boundary to improve understanding of the behavior of material failure under dynamic loading.

In conventional studies of a semi-infinite crack in an unbounded medium subjected to dynamic loading, the complete solution is obtained by integral transform methods together with direct application of the Wiener-Hopf technique (Noble [1]) and the Cagniard-de Hoop method (de Hoop [2]) of Laplace inversion. If the loading is replaced by a nonuniform distribution having a characteristic length, then the same procedure using integral transformation methods does not apply. The problem of an elastic solid containing a semi-infinite crack subjected to concentrated point loading on the faces of the crack has been previously studied by Freund [3]. He proposed a fundamental solution arising from an edge dislocation climbing along the line ahead of the crack tip with a constant speed to overcome these difficulties of the case with a characteristic length. The solution can be constructed by taking an integration over a climbing dislocation of different moving velocity. Basing their procedure on this method, Brock et al. [4-6] and $\mathrm{Ma}$ and Hou [7,8] have analyzed a series problems of a semi-infinite crack subjected to impact loading. Lee and Freund [9] analyzed fracture initiation of an edge cracked plate subjected to an asymmetric impact. For the problem of a crack of finite length subjected to a suddenly applied crack face pressure, the integral transforms can be applied and a relationship among sectionally analytic function is obtained which is somewhat more complicated in form than the standard Wiener-Hopf equations. In principal, the generalized Wiener-Hopf equations can be solved iteratively to obtain the complete solution. In practice, however, only the first step in the iteration process has been carried out by Thau and Lu [10] up until a dilatational wave has traveled the length of the crack twice. A thorough summary of the application of the main direct methods of analysis for transient problem in dynamic fracture for elastic or inelastic problems has been given by Freund [11]. For incident antiplane harmonic wave, Sih and Loeber [12] and Mal [13] made detailed studies on the displacement and the stress field in the vicinity of a finite crack. 
For antiplane problems, many solutions have been derived based on Green's function method. This approach was originally developed for solving problems in potential theory, but it has been applied with great success to solve linear boundary value problems in mathematical physics. The method was used by Kostrov [14] and Achenbach $[15,16]$ to solve the problems of crack propagation. The Green's function represents the displacement for a two-dimensional line source, linear superposition is employed and an integral equation can be constructed from the condition that the displacement vanishes ahead of the crack tip. The region of integration for the integral equation is in a complicated shape, generally being bounded by a hyperbola and a number of straight lines. For points ahead of the crack tip, the region of integration reduces to a triangular region and the stress in the plane of the crack can thus be determined without difficulty. However, for material points not on the crack tip line, the region of integration is very complicated and careful analysis is needed.

Whenever dynamic loading is applied to a body with an internal crack, the resulting stress waves may initiate crack growth. Few solutions for a cracked elastic solid subjected to dynamic loading are available. Exact transient closed form solutions for a stationary semi-infinite crack subjected to a suddenly applied dynamic body force in an unbounded medium have been obtained by Tsai and $\mathrm{Ma}$ [17] for the inplane case and by Ma and Chen [18] and Brock [19] for the antiplane case. Tsai and Ma [20] studied the dynamic response of the stress intensity factor of an inclined semi-infinite crack inside an elastic half-plane and subjected to dynamic impact inplane loading on the half plane. Brock et al. [6] investigated the case in which the crack is normal to the half-plane surface and the point loading is applied to the surface directly above the crack tip, which is a special case of the general formulation by Tsai and Ma [20]. Because of the difficulty for the transient analysis of the crack interaction with half-plane boundary, the few results in this field just mentioned above are valid only before the first wave scattered from the crack tip returns to the crack tip after being reflected by the half-plane boundary. The problem to be considered in this study is the transient response of an elastic half-plane, with a perpendicular crack extending from infinity to a location near the half-plane surface, and dynamic antiplane loadings are applied on the semi-infinite crack faces. The specific dynamic loading to be considered in detail is a pair of point loading (Fig. 1) applied on the crack faces.

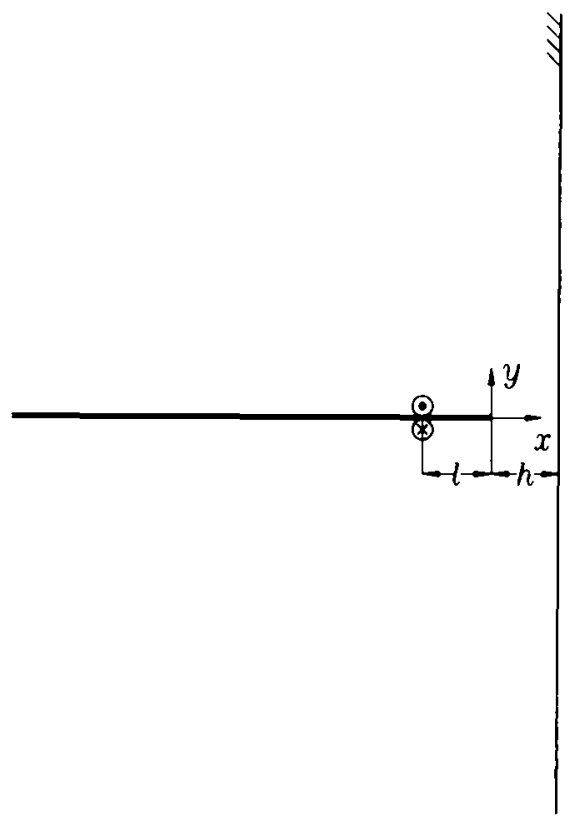

Fig. 1. Geometrical configuration of a subsurface semi-infinite crack subjected to dynamic point loading on the crack face. 
In this study, a free boundary and a semi-infinite crack are incorporated into the analysis which will make the analysis extremely difficult. In analyzing this problem, the refiections and diffractions of stress waves by the half-plane boundary and by the crack will generate an infinite number of waves, must be taken into account. It is impossible to solve this complicated problem by using the standard Wiener-Hopf technique and some other approach must be followed. A new fundamental solution is used for overcoming these difficulties. This alternative fundamental solution is successfully applied towards solving the problem and is to be demonstrated as an efficient methodology. The final formulations for stress intensity factors are expressed explicitly and the dynamic effect of each wave is presented in a closed form. The results are valid for an infinite number of waves that are scattered from the crack tip and reflected by the free boundary.

\section{REQUIRED FUNDAMENTAL SOLUTIONS}

As usual in problems of the type considered here, superposition of solutions plays a significant role. The solutions of the problem considered in this study can be determined by superposition of the following problems. Problem A treats a dynamic distributed traction acting on semi-infinite crack faces in an unbounded medium, which induces a traction on the plane that will eventually define the half-plane boundary. In problem $B$, a semi-infinite half-plane is considered in which the boundary is subjected to tractions that are equal and opposite to those on the corresponding planes in problem A. Problem $\mathrm{C}$ considers an infinite body containing a semi-infinite crack in which the crack face is subjected to the waves which are generated by the half-plane boundary in problem $B$. The reflected waves induced from the half-plane boundary as indicated in problem B, can be obtained by employing the method of images.

Problem $\mathrm{C}$ in the aforementioned three fundamental problems is the only one that needs careful analysis. For most of the dynamic problems, the propagating waves can be represented in an exponential functional form in the Laplace transform domain of time. The reflected and diffracted waves generated by the half-plane boundary and by the crack can thus be constructed by the superposition method; that is, if the responses toward an applied exponentially distributed traction on boundaries in the Laplace transform domain can be obtained preliminarily. The superposition scheme proposed in this study, unlike usual superposition methods which are performed in the time domain, is performed in the Laplace transform domain.

Consider the fundamental problem of antiplane deformation for a semi-infinite crack contained in an unbounded medium. The solution for an exponentially distributed loading applied at the crack faces in the Laplace transform domain will be referred to as the fundamental solution. The problem can be viewed as a half-plane problem with the material occupying the region $y \geq 0$, subject to the following mixed boundary conditions in the Laplace transform domain

$$
\begin{array}{cc}
\bar{\tau}_{y z}(x, 0, p)=\mathrm{e}^{p \eta x} & -x<x<0, \\
\bar{w}(x, 0, p)=0 & 0<x<x,
\end{array}
$$

where $p$ is the Laplace transform parameter and $\eta$ is a constant. The overbar symbol is used for denoting the transform on time $t$. This fundamental problem can be solved by using the standard transform method and the Wiener-Hopf technique. The governing equation can be represented by the two-dimensional wave equation

$$
\frac{\partial^{2} w}{\partial x^{2}}+\frac{\partial^{2} w}{\partial y^{2}}=b^{2} \frac{\partial^{2} w}{\partial t^{2}}
$$

where $b$ is the slowness of the shear wave given by

$$
b=1 / v=\sqrt{\rho / \mu},
$$


in which $w(x, y, t)$ is the displacement normal to the $x y$-plane; $v$ is the shear wave speed, $\mu$ and $\rho$ are the respective shear modulus and the mass density of the material. The nonvanishing shear stresses are

$$
\tau_{y z}=\mu \frac{\partial w}{\partial y}, \quad \tau_{x z}=\mu \frac{\partial w}{\partial x} .
$$

This fundamental problem can be solved by the application of integral transforms. Applying the one-sided Laplace transform over time, the two-sided Laplace transform over $x$ under the restriction of $\operatorname{Re}(\eta)>\operatorname{Re}(\lambda)$, finally the Wiener-Hopf technique is implemented. The solutions of stresses and displacement for the boundary conditions (2.1) and (2.2) in the transform domain are

$$
\begin{gathered}
\bar{\tau}_{y z}(x, y, p)=\frac{1}{2 \pi i} \int_{\Gamma_{\lambda}} \frac{(b+\lambda)^{1 / 2} \mathrm{e}^{p(\alpha y-\lambda x)}}{(b+\eta)^{1 / 2}(\eta-\lambda)} \mathrm{d} \lambda, \\
\bar{\tau}_{A z}(x, y, p)=-\frac{1}{2 \pi i} \int_{\Gamma_{\lambda}} \frac{\lambda \mathrm{e}^{p(\alpha y-\lambda, \lambda)}}{(b+\eta)^{1 / 2}(\eta-\lambda)(b-\lambda)^{1 / 2}} \mathrm{~d} \lambda, \\
\bar{w}(x, y, p)=-\frac{1}{2 \pi i} \int_{\Gamma_{\lambda}} \frac{\mathrm{e}^{-p(\alpha y-\lambda x)}}{\mu p(b+\eta)^{1 / 2}(\eta-\lambda)(b-\lambda)^{1 / 2}} \mathrm{~d} \lambda,
\end{gathered}
$$

where

$$
\alpha=\left(b^{2}-\lambda^{2}\right)^{1 / 2} .
$$

The corresponding result of the dynamic stress intensity factor in the Laplace transform domain is

$$
\begin{aligned}
\bar{K}(p) & =\lim _{x \rightarrow 0} \sqrt{2 \pi x} \bar{\tau}_{y z}(x, 0, p) \\
& =-\frac{\sqrt{2}}{\sqrt{p}(b+\eta)^{1 / 2}} .
\end{aligned}
$$

\section{DYNAMIC STRESS INTENSITY FACTOR FOR APPLYING POINT LOADING}

In this section, a semi-infinite crack subjected to dynamic concentrated loadings acting at a finite distance $l$ from the crack tip is investigated in detail. We will focus our attention on the solution for the dynamic stress intensity factor. Consider a stress-free homogeneous half-plane medium containing a semi-infinite crack which is perpendicular to the surface of the half-plane. At time $t=0$, a pair of concentrated dynamic loading of magnitude $\sigma_{0}$ acts at $x=-l$ on each face of the crack as shown in Fig. 1. The time dependence of the loading is represented by the Heaviside step function $H(t)$. This problem has a characteristic length and a direct attempt towards solving this problem by transform and Wiener-Hopf techniques is not applicable. The transient elastodynamic problem is solved by superposition of the fundamental solutions obtained in the previous section in the Laplace transform domain. The transient solutions are composed of an incident field, reflected field and diffracted field which are denoted by superscripts of $i, r$ and $d$, respectively. The incident field generated by applying point loading can be expressed in the Laplace transform domain as follows

$$
\bar{\tau}_{y z}^{i}(x, 0, p)=-\frac{1}{2 \pi i} \int_{r_{i}} \sigma_{0} \mathrm{e}^{p \lambda(x+1)} \mathrm{d} \lambda .
$$

When we combine equations (2.5) and (3.1), the solution of diffracted wave is

$$
\bar{\tau}_{y z}^{\prime}(x, y, p)=\frac{1}{4 \pi^{2}} \int_{\Gamma_{\eta_{1}}} \int_{\Gamma_{\eta_{2}}} \frac{\sigma_{0}\left(b+\eta_{2}\right)^{1 / 2}}{\left(b+\eta_{1}\right)^{1 / 2}\left(\eta_{1}-\eta_{2}\right)} \mathrm{e}^{\rho \eta_{1}^{\prime}} \mathrm{e}^{-p\left(\alpha y-\eta_{2} x\right)} \mathrm{d} \eta_{2} \mathrm{~d} \eta_{1} .
$$


We carry out the inverse transformation by using the Cagniard-de Hoop technique, the incident and diffracted fields in time domain are represented as follows

$$
\begin{aligned}
\tau_{y z}^{i+d}(x, y, t)=-\frac{\sigma_{0} t}{\sin \theta_{2} H\left(t-b r_{2}\right)} & \\
\pi r_{2}\left(t^{2}-b^{2} r_{2}^{2}\right)^{1 / 2} & \\
& \quad+\frac{\sigma_{0}}{2 \pi^{2}} \int_{b l}^{t-b r} \operatorname{Re}\left[G\left(\eta_{1}^{+}, \eta_{2}^{+}\right) \frac{\partial \eta_{1}^{+}}{\partial t_{1}} \frac{\partial \eta_{2}^{+}}{\partial t_{2}}-G\left(\eta_{1}^{-}, \eta_{2}^{+}\right) \frac{\partial \eta_{1}^{-}}{\partial t_{1}} \frac{\partial \eta_{2}^{+}}{\partial t_{2}}\right] \mathrm{d} t_{1},
\end{aligned}
$$

where

$$
\begin{gathered}
\eta_{1}^{+}=-\frac{t_{1}}{l} \pm i \varepsilon, \\
\eta_{2}^{ \pm}=-\frac{t_{2} \cos \theta}{r} \pm i \frac{\sin \theta}{r} \sqrt{t_{2}^{2}-b^{2} r^{2}} \\
r_{2}=\left((x-l)^{2}+y^{2}\right)^{1 / 2}, \quad \theta_{2}=\cos ^{-1}\left(\frac{x-l}{r_{2}}\right), \\
t_{1}+t_{2}=t .
\end{gathered}
$$

The first term presented in (3.3) is the solution for incident wave. The second term represents the diffracted field which can be integrated and the result is exactly the static full field solution for applied point loading at the crack faces plus the solution for incident wave but with minus sign. Hence, at time $b l<t<b(l+h)$, it can be concluded that the transient shear stress $\tau_{y z}^{i+d}$ due to the contributions from incident and diffracted waves jumps from the dynamic transient solution to the appropriate static value instantaneously upon arrival of the diffracted $d$ wave. Hence the solution becomes

$$
\tau_{y z}^{i+d}(x, y, t)=\frac{\sigma_{0}}{\pi} \sqrt{\frac{l}{r}} \frac{r \cos (3 \theta / 2)+l \cos (\theta / 2)}{r^{2}+2 r l \cos \theta+l^{2}} H(t-b(l+r)) .
$$

The numerical calculation for (3.3) is performed and the result is shown in Fig. 2. It is shown very clearly in this figure that the full field solution of shear stress jumps from transient solution to the correspondent static solution expressed in (3.4) for a semi-infinite crack in an unbounded medium. The correspondent dynamic stress intensity factor is

$$
K^{d}(t)=\sigma_{0} \sqrt{\frac{2}{\pi l}} H(t-b l) .
$$

The interesting result of (3.5) is that the dynamic stress intensity factor jumps from zero to the

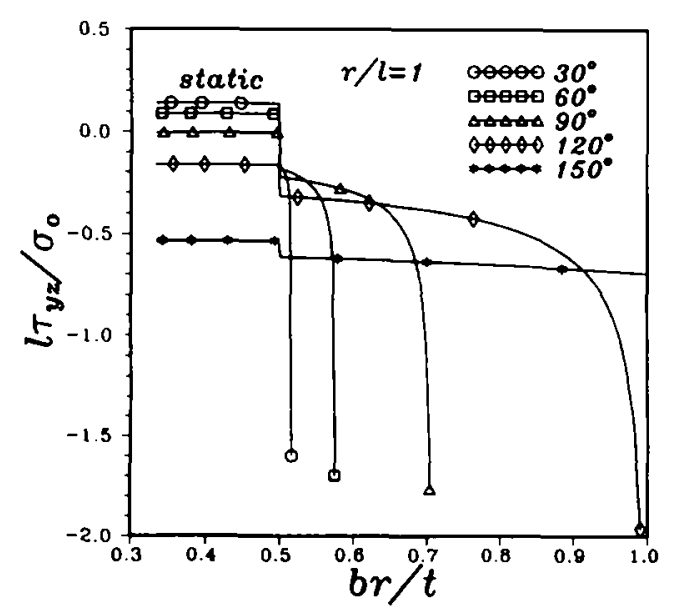

Fig. 2. The full field transient stress response for applying dynamic point loading on the crack face at time $b l<t<b(l+h)$. 
static value for a semi-infinite crack in an unbounded medium after the incident wave generated from the loading point arrives at the crack tip. After some later time, $(t>b(l+h))$, the diffracted $d$ wave will be reflected from the half-plane surface which will be denoted as the $d r$ wave. The solution for $d r$ wave in the Laplace transform domain is

$$
\bar{\tau}_{y z}^{d r}(x, y, p)=\frac{1}{4 \pi^{2}} \int_{\Gamma_{\eta_{1}}} \int_{\Gamma_{\eta_{z}}} \frac{\sigma_{0}\left(b+\eta_{2}\right)^{1 / 2}}{\left(b+\eta_{1}\right)^{1 / 2}\left(\eta_{1}-\eta_{2}\right)} \mathrm{e}^{\rho \eta_{1} \prime} \mathrm{e}^{-p\left(x y+\eta_{2}(x-2 h) \mid\right.} \mathrm{d} \eta_{2} \mathrm{~d} \eta_{1} .
$$

The reflected $d r$ wave will arrive at the crack tip at a later time and a diffracted $d r d$ wave will be induced. The solution for $d r d$ wave can be constructed from (2.5) and (3.6), the result expressed in the transform domain is

$$
\begin{aligned}
& \bar{\tau}_{y z}^{d r d}(x, y, p)=\frac{1}{(2 \pi i)^{3}} \int_{\Gamma_{\eta_{1}}} \int_{\Gamma_{\eta_{2}}} \int_{\Gamma_{\eta_{3}}} \frac{\sigma_{0}\left(b-\eta_{2}\right)^{1 / 2}\left(b+\eta_{3}\right)^{1 / 2}}{\left(b+\eta_{1}\right)^{1 / 2}\left(\eta_{1}+\eta_{2}\right)\left(b+\eta_{2}\right)^{1 / 2}\left(\eta_{2}-\eta_{3}\right)} \mathrm{e}^{p\left(\eta_{1} / \cdot 2 \eta_{2} h\right)} \\
& e^{p\left(\alpha y-\eta_{2} x\right)} \mathrm{d} \eta_{3} \mathrm{~d} \eta_{2} \mathrm{~d} \eta_{1} \text {. }
\end{aligned}
$$

The corresponding stress intensity factor in the Laplace transform domain can be obtained from (2.8) and (3.6)

$$
\bar{K}^{d r d}(p)=\frac{1}{4 \pi^{2}} \int_{\Gamma_{\eta_{1}}} \int_{\Gamma_{\eta_{2}}} \frac{\sigma_{0} \sqrt{2} \sqrt{b-\eta_{2}}}{\sqrt{p} \sqrt{b+\eta_{1}}\left(\eta_{1}+\eta_{2}\right) \sqrt{b+\eta_{2}}} \mathrm{e}^{p \eta_{1}^{\prime}} \mathrm{e}^{-2 p \eta_{2} h} \mathrm{~d} \eta_{2} \mathrm{~d} \eta_{1} .
$$

The dynamic stress intensity factor in time domain will be

$$
K^{d r d}(t)=\frac{\sigma_{0}}{\pi^{2}} \int_{b l+2 h h}^{t} \int_{h l}^{t-2 h h} \frac{\sqrt{2 l} \sqrt{\tau-t_{1}-2 b h}}{\sqrt{\pi} \sqrt{(t-\tau)\left(t_{1}-b l\right)\left(\tau-t_{1}+2 b h\right)}\left[\left(\tau-t_{1}\right) l-2 h t_{1}\right.} \mathrm{d} t_{1} \mathrm{~d} \tau .
$$

The associated wave fronts for a short time period are shown in Fig. 3. Follow the similar procedure, the complete transient solution for dynamic stress intensity factor can be obtained explicitly and can be simplified into a very compact form as follows

$$
K(t)=K^{d .1}(t)+\sum_{n=2}^{\infty} K^{d . n}(t)
$$

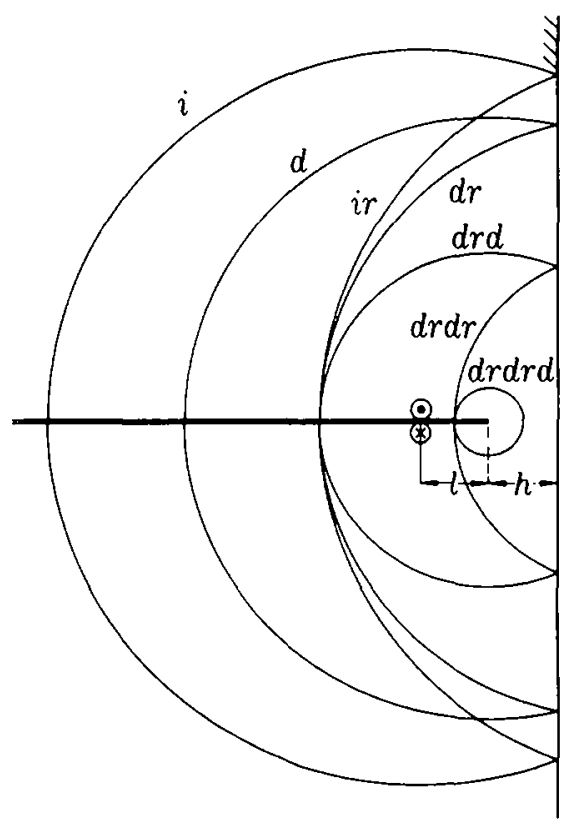

Fig. 3. Wave fronts of the incident, reflected and diffracted waves for a short time period after the dynamic point loading is applied on the crack face. 
where

$$
\begin{gathered}
K^{d, 1}(t)=\sigma_{0} \sqrt{\frac{2}{\pi l}} H(t-b l), \\
K^{d, n}(t)=\frac{\sigma_{0}}{\pi^{n}} \int_{b l+2(n-1) b h}^{t} \int_{b l}^{b_{1}} \int_{2 b h}^{b_{2}} \ldots \int_{2 b h}^{b_{n-1}} \\
\quad \times \frac{\sqrt{2 l} \sqrt{\left(t_{2}-2 b h\right)\left(t_{3}-2 b h\right) \ldots\left(t_{n}-2 b h\right)}}{\sqrt{\pi} \sqrt{(t-\tau)\left(t_{1}-b l\right)} \sqrt{\left(t_{2}+2 b h\right)\left(t_{3}+2 b h\right) \ldots\left(t_{n}+2 b h\right)}} \\
\times \frac{1}{\left(t_{2} l-2 t_{1} h\right)\left(t_{2}+t_{3}\right)\left(t_{3}+t_{4}\right) \ldots\left(t_{n-1}+t_{n}\right)} \mathrm{d} t_{n-1} \mathrm{~d} t_{n-2} \ldots \mathrm{d} t_{1} \mathrm{~d} \tau, n=2,3,4, \ldots,
\end{gathered}
$$

in which

$$
\begin{gathered}
b_{1}=\tau-2(n-1) b h, \\
b_{m+1}=\tau-t_{1}-t_{2}-\ldots-t_{m}-2(n-m-1) b h, \quad m=1,2,3, \ldots, n-1, \\
t_{j}=\tau-t_{1}-t_{2}-\ldots-t_{j-1}, \quad j=2,3,4, \ldots
\end{gathered}
$$

The corresponding static solution of the stress intensity factor for this problem is

$$
K^{s}=\frac{2(h+l) \sigma_{0}}{\sqrt{\pi h(l+2 h)}} .
$$

The transient response of the dynamic stress intensity factor is shown in Fig. 4 and the solution is normalized by the static value expressed in (3.12). Up to this point, the time dependency of the point loading profile is a simple step function in time. However, the solution for spatially distributed traction on crack faces or for more general time dependence can be obtained by superposition of the point loading solution.

There is another way to construct the transient solution and more physical insights will be shown. We have already pointed out previously in this section that at time $b l<t<b(l+h)$, the static field for a semi-infinite crack in an unbounded medium subjected to a static point loading is radiated out for the full field behind the diffracted $d$ wave front. The correspondent numerical result is shown in Fig. 2. The stress at any point along the crack line jumps from zero to a static value instantaneously after the diffracted $d$ wave (or incident $i$ wave) has passed this point. The result is

$$
\tau_{y z}^{i+d}(x, 0, t)=\frac{\sigma_{0}}{\pi} \sqrt{\frac{l}{x}} \frac{1}{x+l} H(t-b(x+l)) .
$$

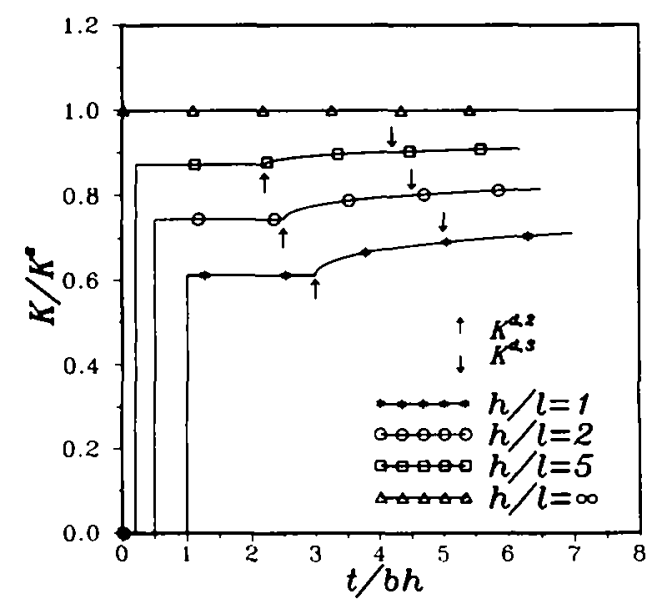

Fig. 4. Transient response of the dynamic stress intensity factor for applying a dynamic point loading on the crack face. 
At time $b(l+h)<t<b(l+2 h)$, the reflected $d r$ wave generated from the free surface propagates toward the crack and another static solution will be developed behind the $d r$ wave front, the result for the stress along the crack line due to $d r$ wave becomes

$$
\tau_{y z}^{d r}(x, 0, t)=-\frac{\sigma_{0}}{\pi} \sqrt{\frac{l}{2 h-x}} \frac{1}{2 h-x+l} H(t-b(l+2 h-x)),
$$

which corresponds to the appropriate static solution of a semi-infinite crack located at $x=2 h$ and the solution can be constructed from the method of image.

At time $b(l+2 h)<t<b(l+3 h)$, a diffracted $d r d$ wave will be generated from the crack tip. Although the diffracted $d r d$ wave has been expressed in (3.7) in a very complicated form, it can also be constructed by a more simple way from the important phenomenon that a static field is developed behind the $d r$ wave front. The stress induced by $d r d$ wave can then be determined by analyzing the situation in which equal but opposite tractions are applied to the faces of the crack by $d r$ wave. The stress along the crack line induced by $d r d$ wave can be obtained by integrating a static loading shown in (3.14) and the static Green function in (3.13). We have

$$
\tau_{y z}^{d r d}(x, 0, t)=\frac{\sigma_{0}}{\pi^{2}} \int_{0}^{(t / h \cdots 1-2 h-x) / 2} \sqrt{\frac{l}{2 h+\xi}} \frac{1}{2 h+\xi+l} \sqrt{\frac{\xi}{x}} \frac{1}{x+\xi} \mathrm{d} \xi .
$$

Hence the transient solution for point loading can be constructed simply by superposition of the well known static Green's function for a semi-infinite crack in an unbounded medium. The transient response of stress $\tau_{y z}$ along the crack line is shown in Fig. 5. It shows that the stress will take on a appropriate static value instantaneously upon arrival of the $d$ wave and this static value is maintained before the $d r$ wave arrives. After the $d r$ wave has passed, the stress jumps to another static value until the $d r d$ wave reaches the field point.

The correspondent dynamic stress intensity factor can also be obtained by a similar way, the result is

$$
\begin{aligned}
K^{d r d}(t) & =\frac{\sigma_{0}}{\pi} \int_{0}^{(t / b-1-2 h) / 2} \sqrt{\frac{l}{2 h+\xi}} \frac{1}{2 h+l+\xi} \sqrt{\frac{2}{\pi \xi}} \mathrm{d} \xi \\
= & \frac{\sigma_{0}}{\pi} \sqrt{\frac{2}{\pi(l+2 h)} \ln \left[\frac{h(t / b+l+2 h)}{2 h^{2}+(t / b-l)(l+h)-\sqrt{l(l+2 h)(t / b-l-2 h)(t / b-l+2 h)}}\right] .}
\end{aligned}
$$

The expression in (3.16) is the same as the solution presented in (3.9) with two integrations, which has already been checked out by numerical calculation.

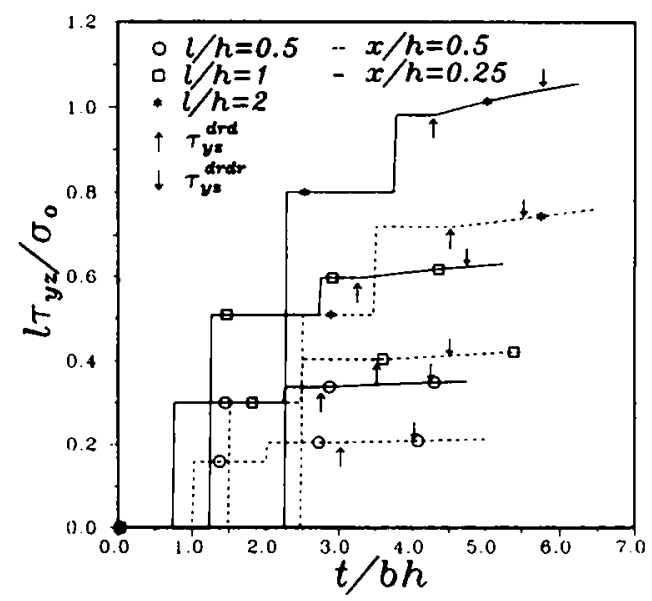

Fig. 5. Transient shear stresses for the field point along the crack line due to applying point loading on the crack face. 
Table 1. Comparison of the numerical results for $K^{\text {drd }}$ from integration of the solution of point loading and the solution of applying uniform loading on the crack faces

\begin{tabular}{ccc}
\hline$t / b h$ & Uniform loading & Green function \\
\hline 2.0 & $0.00000 \times 10^{\prime \prime}$ & $0.00000 \times 10^{\prime \prime}$ \\
2.2 & $0.18779 \times 10^{2}$ & $0.18778 \times 10^{-2}$ \\
2.4 & $0.70990 \times 10^{2}$ & $0.70987 \times 10^{-2}$ \\
2.6 & $0.15146 \times 10^{-1}$ & $0.15145 \times 10^{-1}$ \\
2.8 & $0.25607 \times 10^{-1}$ & $0.25606 \times 10^{-1}$ \\
3.0 & $0.38150 \times 10^{-1}$ & $0.38148 \times 10^{1}$ \\
3.2 & $0.52502 \times 10^{-1}$ & $0.52503 \times 10^{-1}$ \\
3.4 & $0.68437 \times 10^{-1}$ & $0.68440 \times 10^{-1}$ \\
3.6 & $0.85767 \times 10^{1}$ & $0.85770 \times 10^{-1}$ \\
3.8 & $0.10433 \times 10^{\prime \prime}$ & $0.10433 \times 10^{-1}$ \\
4.0 & $0.12399 \times 10^{\prime \prime}$ & $0.12399 \times 10^{\prime \prime}$ \\
4.2 & $0.14463 \times 10^{\prime \prime}$ & $0.14464 \times 10^{\prime \prime}$ \\
4.4 & $0.16615 \times 10^{\prime \prime}$ & $0.16616 \times 10^{\prime \prime}$ \\
4.6 & $0.18846 \times 10^{\prime \prime}$ & $0.18846 \times 10^{\prime \prime}$ \\
4.8 & $0.21149 \times 10^{\prime \prime}$ & $0.21149 \times 10^{\prime \prime}$ \\
5.0 & $0.23516 \times 10^{\prime \prime}$ & $0.23516 \times 10^{\prime \prime}$ \\
5.2 & $0.25941 \times 10^{\prime \prime}$ & $0.25941 \times 10^{\prime \prime}$ \\
5.4 & $0.28420 \times 10^{\prime \prime}$ & $0.28420 \times 10^{\prime \prime}$ \\
5.6 & $0.30947 \times 10^{\prime \prime}$ & $0.30947 \times 10^{\prime \prime}$ \\
5.8 & $0.33518 \times 10^{\prime \prime}$ & $0.33518 \times 10^{\prime \prime}$ \\
6.0 & $0.36130 \times 10^{\prime \prime}$ & $0.36130 \times 10^{\prime \prime}$ \\
\hline
\end{tabular}

The problem of applying point loading on crack faces analyzed in this section is the Green's solution of the semi-infinite crack. This solution can be used to construct solutions for arbitrary crack face loadings by superposition. Hence, the solution for uniformly distributed loading applied on the crack faces can be obtained by integration of (3.16), the result is

$$
K_{\text {uniform }}^{\text {drd }}(t)=\frac{\sigma_{0}}{\pi} \sqrt{\frac{2}{\pi}} \int_{0}^{x} \int_{0}^{(t / h-\eta-2 h) / 2} \frac{\sqrt{\eta}}{\sqrt{\xi(\xi+2 h)}(\xi+\eta+2 h)} \mathrm{d} \xi \mathrm{d} \eta .
$$

The numerical result of $K^{d r d}$ in (3.17) is compared with the solution of applying a uniformly distributed loading on crack faces, these two numerical results are in excellent agreement as indicated in Table 1.

In a similar procedure, at time $t>b(l+4 h)$ the diffracted $d r d r d$ wave will be radiated out from the crack tip and the induced dynamic stress intensity factor is

$$
\begin{aligned}
K^{d r d r d}(t)=\frac{\sigma_{0}}{\pi^{2}} \sqrt{\frac{2 l}{\pi}} \int_{0}^{(l / b-1-4 h) / 2} \int_{0}^{(1 / h-1-4 h-2 \eta) / 2} & \\
& \times \frac{\sqrt{\eta}}{\sqrt{\xi(\eta+2 h)(\xi+2 h)}} \frac{1}{(\eta+2 h+l)(\xi+\eta+2 h)} \mathrm{d} \xi \mathrm{d} \eta .
\end{aligned}
$$

The solution of applying uniformly distributed loading on the crack faces can be obtained from the point loading solution expressed in (3.18) as follows

$$
\begin{aligned}
K_{\text {uniform }}^{d r d r d}(t)=\frac{\sigma_{0}}{\pi^{2}} \sqrt{\frac{2}{\pi}} \int_{0}^{\infty} \int_{0}^{(t / h-\xi-4 h) / 2} & \int_{b}^{(t / b-\zeta-4 h-2 \eta) / 2} \\
& \times \frac{\sqrt{\eta \zeta}}{\sqrt{\xi(\eta+2 h)(\xi+2 h)}} \frac{1}{(\eta+\zeta+2 h)(\xi+\eta+2 h)} \mathrm{d} \xi \mathrm{d} \eta \mathrm{d} \zeta .
\end{aligned}
$$

The solution expressed in (3.19) is also compared numerically with the solution for 
Table 2. Comparison of the numerical results for $K^{\text {drtrat }}$ from integration of the solution of point loading and the solution of applying uniform loading on the crack faces

\begin{tabular}{ccc}
\hline$l / b h$ & Uniform loading & Grecn function \\
\hline 4.0 & $0.00000 \times 10^{6}$ & $0.00000 \times 10^{\prime \prime}$ \\
4.2 & $0.99725 \times 10^{\circ}$ & $0.99672 \times 10^{-6}$ \\
4.4 & $0.10438 \times 10^{-4}$ & $0.10436 \times 10^{4}$ \\
4.6 & $0.40049 \times 10^{4}$ & $0.40044 \times 10^{-4}$ \\
4.8 & $0.10205 \times 10^{3}$ & $0.10204 \times 10^{3}$ \\
5.0 & $0.20801 \times 10^{3}$ & $0.20800 \times 10^{3}$ \\
5.2 & $0.36847 \times 10^{3}$ & $0.36849 \times 10^{-3}$ \\
5.4 & $0.59276 \times 10^{3}$ & $0.59291 \times 10^{3}$ \\
5.6 & $0.88906 \times 10^{3}$ & $0.88941 \times 10^{3}$ \\
5.8 & $0.12644 \times 10^{2}$ & $0.12650 \times 10^{-2}$ \\
6.0 & $0.17250 \times 10^{2}$ & $0.17259 \times 10^{-2}$ \\
6.2 & $0.22757 \times 10^{-2}$ & $0.22770 \times 10^{2}$ \\
6.4 & $0.29211 \times 10^{2}$ & $0.29227 \times 10^{-2}$ \\
6.6 & $0.36646 \times 10^{-2}$ & $0.36666 \times 10^{2}$ \\
6.8 & $0.45093 \times 10^{2}$ & $0.45116 \times 10^{2}$ \\
7.0 & $0.54574 \times 10^{2}$ & $0.54612 \times 10^{2}$ \\
7.2 & $0.65109 \times 10^{2}$ & $0.65141 \times 10^{2}$ \\
7.4 & $0.76712 \times 10^{-2}$ & $0.76749 \times 10^{2}$ \\
7.6 & $0.89396 \times 10^{2}$ & $0.89436 \times 10^{2}$ \\
7.8 & $0.10316 \times 10^{-1}$ & $0.10321 \times 10^{\prime}$ \\
8.0 & $0.11803 \times 10^{1}$ & $0.11808 \times 10^{1}$ \\
\hline
\end{tabular}

uniformly distributed loading, the results are shown in Table 2. Good agreement of these two expressions for the numerical results is also indicated.

\section{CONCLUSIONS}

Most of the problems that have been studied in the development of fracture mechanics are quasi-static. Because of loading conditions and material properties, numerous problems have existed for which the assumption of quasi-static deformation is invalid and the inertia of the material must be taken into account. It would be difficult to state precise conditions under which inertia effects might be neglected. The propagation of stress waves through an unbounded medium is not a difficult subject. However, if boundaries are introduced, reflected and diffracted waves will be generated from boundaries, making the problem much more complicated.

The transient response of a half-space containing a perpendicular semi-infinite crack has been considered here to gain understanding of the interaction of stress waves with material defects. This problem contains a characteristic length and is solved by superposition of a proposed fundamental solution in the Laplace transform domain. The exact transient solutions for dynamic stress intensity factors over a long period of time are obtained in this study. The complicated closed form transient solutions are expressed in a very simple and compact formulation that account for all contributions coming from reflected and diffracted waves. The transient stresses near the crack tip can be used for analyzing the condition for unstable crack propagation. It is shown in the numerical result that the major contribution of the stress intensity factor is due to the influence of the first few waves.

A pair of concentrated loadings applied on the crack faces is investigated in detail. The solution for point loading can be considered as the Green function for the associated problems, the solution for any arbitrary spatially distributed loading or more general time dependence can be obtained by superposition. The transient solution for applied point loading has many interesting phenomena. We find that the static field for a semi-infinite crack in an unbounded medium subjected to a static point loading is radiated out for the full field behind the first 
diffracted wave ( $d$ wave) front. The stress along the crack line jump to a static value after the first diffracted wave has passed and jump to another static value after the first reflected wave ( $d r$ wave) generated from the half-plane boundary has arrived. The contribution for the second diffracted wave ( $d r d$ wave) can be constructed by superposition of the static solution for applying a static point loading on a semi-infinite crack face in an unbounded medium and the stress induced by the first reflected wave on the crack face. Hence, the transient solution for dynamic point loading can be constructed simply by superposition of the well known static Green's function for a semi-infinite crack in an unbounded medium. The transient solution for dynamic point loading is then used to construct the transient solution for uniformly distributed loading. Numerical results for dynamic stress intensity factor from integration of the solution of point loading and the solution of applying uniform loading are in excellent agreement.

Acknowledgement-The research support of the National Science Council. Republic of China, through Grant NSC 81-0401-E002-18 at National Taiwan University, is gratefully acknowledged.

\section{REFERENCES}

[1] B. NOBLE, The Wiener-Hopf Technique. Pergamon Press, New York (1958).

[2] A. T. DE HOOP, Representation theorems for the displacement in an elastic solid and their application to elastodynamic diffraction theory. Doctoral dissertation, Technische hoegschool, Delft (1958).

[3] L. B. FREUND, Int. J. Engng Sci. 12, 179 (1974).

[4] L. M. BROCK, Int. J. Solids Struct. 18, 467 (1982).

[5] L. M. BROCK, J. Elast. 14, 415 (1984).

[6] L. M. BROCK, M. JOLLES and M. SCHROEDL, J. Appl. Mech. 52, 287 (1985).

[7] C. C. MA and Y. C. HOU, Int. J. Engng Sci. 28, 1321 (1990).

[8] C. C. MA and Y. C. HOU, J. Appl. Mech. 58, 703 (1991).

[9] Y. J. LEE and L. B. FREUND, J. Appl. Mech. 57, 104 (1990).

[10] S. A. THAU and T. H. LU, Int. J. Solids Struct. 7, 731 (1971).

[11] L. B. FREUND, Dynamic Fracture Mechanics. Cambridge University Press, Cambridge (1990).

[12] G. C. SIH and J. F. LOEBER, Q. Appl. Math. 27, 193 (1969).

[13] A. K. MAL, Int. J. Engng Sci. 8, 763 (1970).

[14] B. V. KOSTROV, Prikl. Mat. Mekh. 30, 1241 (1966).

[15] J. D. ACHENBACH, Int. J. Engng Sci. 8, 947 (1970).

[16] J. D. ACHENBACH, Z. Angew. Math. Phys. 21, 887 (1970).

[17] C. H. TSAI and C. C. MA, J. Appl. Mech. 59, 804 (1992).

[18] C. C. MA and S. K. CHEN, Wave Motion 17, 161 (1993).

[19] L. M. BROCK, Q. Appl. Math. XLIV, 265 (1986).

[20] C. H. TSAI and C. C. MA, Int. J. Solids Struct. 30, 2163 (1993). 